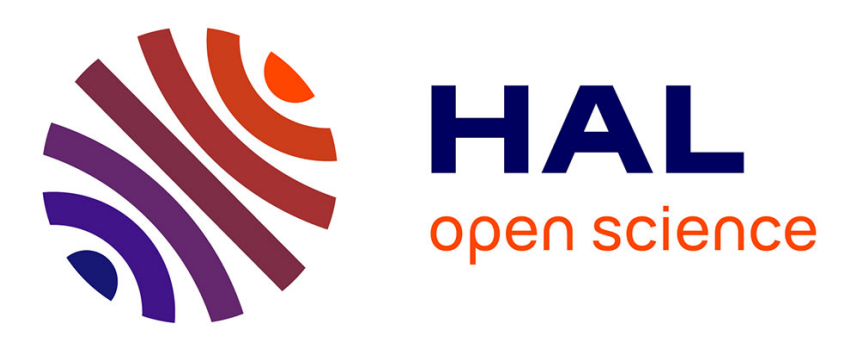

\title{
Modelling of phase transformation kinetics in Ti alloys - Isothermal treatments
}

\author{
Benoît Appolaire, Ludovic Héricher, Elisabeth Aeby-Gautier
}

\section{To cite this version:}

Benoît Appolaire, Ludovic Héricher, Elisabeth Aeby-Gautier. Modelling of phase transformation kinetics in $\mathrm{Ti}$ alloys - Isothermal treatments. Acta Materialia, 2005, 53, pp.3001. 10.1016/j.actamat.2005.03.014 . hal-00163441

\section{HAL Id: hal-00163441 \\ https://hal.science/hal-00163441}

Submitted on 17 Jul 2007

HAL is a multi-disciplinary open access archive for the deposit and dissemination of scientific research documents, whether they are published or not. The documents may come from teaching and research institutions in France or abroad, or from public or private research centers.
L'archive ouverte pluridisciplinaire HAL, est destinée au dépôt et à la diffusion de documents scientifiques de niveau recherche, publiés ou non, émanant des établissements d'enseignement et de recherche français ou étrangers, des laboratoires publics ou privés. 


\title{
Modelling of phase transformation kinetics in $\mathrm{Ti}$ alloys - Isothermal treatments
}

\author{
Benoît Appolaire ${ }^{1}$, Ludovic Héricher, Elisabeth Aeby-Gautier \\ LSG2M, École des Mines de Nancy, Parc de Saurupt, 54042 NANCY Cedex,
} France

\begin{abstract}
We have developed a model which is able to describe the formation of high temperature microstructures in $\beta$ metastable titanium alloys. It is based on the classical theory of nucleation, and on growth laws obtained by assuming that the processes are controlled by the diffusion of the alloying chemical species only. Two morphologies are described: grain boundary allotriomorphs, and Widmanstätten plates gathered in colonies growing from the allotriomorphs. Moreover, a statistical description of the nucleation conditions on $\beta$ grain boundaries has been introduced in order to obtain the global kinetics of the transformation. The model predictions proved to be in good agreement with experimental results.
\end{abstract}

Key words: titanium, modelling, diffusive transformations, nucleation \& growth laws

\section{Introduction}

The final microstructures of titanium alloys are designed through complex thermo-mechanical treatments. In order to predict the phase transformation kinetics and the amount of $\alpha$ phase with different morphologies, it is necessary to model the different successive transformations taking the temperature history into account. Moreover, the deformation occurring prior to the transformation has to be considered too [1].

In this study, we present a model where the transformation kinetics is described by nucleation and growth laws specific to the different morphologies,

$\overline{1}$ Corresponding author. Fax: (33) (0)3 835840 56. e-mail: appolair@mines.inplnancy.fr 
which is potentially able to deal with the effects of a prior deformation. We have applied this model to a multicomponent metastable $\beta$ titanium alloy where one can observe at high temperatures (i.e. $>700{ }^{\circ} \mathrm{C}$ ) the formation of grain boundaries allotriomorphs denoted $\alpha_{\mathrm{GB}}$, and Widmanstätten plates colonies denoted $\alpha_{\mathrm{WGB}}$, shown in Fig. 1.
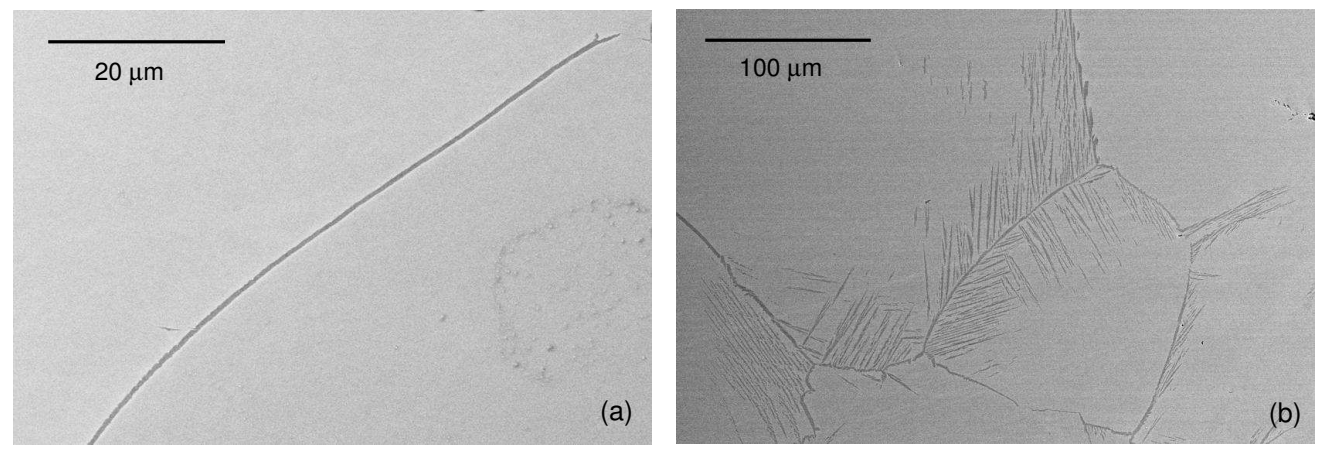

Fig. 1. SEM images of the $\alpha$ phase morphologies observed at $790^{\circ} \mathrm{C}$ in $\beta$-Cez alloy: (a) $\alpha_{\mathrm{GB}}$ and (b) $\alpha_{\mathrm{WGB}}$ colonies growing from $\alpha_{\mathrm{GB}}$.

In section 2, we present the thermodynamic and kinetic data specific to the titanium alloys studied and necessary as inputs to the model. Then, the physical laws describing the nucleation and growth of the different morphologies observed are explained in section 3 . The variation in the nucleation conditions due to the polycrystallinity of the material is taken into account when describing the global phase transformation kinetics in section 4. Finally, the kinetics of the global transformation as well as of some microstructural parameters are discussed in section 5 .

\section{Thermodynamic and kinetic data}

The first requisite for modelling the phase transformation kinetics is a good description of the thermo-chemical equilibrium, i.e. the composition of the $\alpha$ and $\beta$ phases and the corresponding tie-lines as a function of temperature. The equilibrium compositions have been obtained from samples heat treated at different temperatures for durations sufficient to achieve equilibrium in the $\beta$-Cez alloy [2], the composition of which is reported in Table 1.

Table 1

Composition of the so-called $\beta$-Cez titanium alloy.

\begin{tabular}{cccccccc}
\hline Species & $\mathrm{Ti}$ & $\mathrm{Al}$ & $\mathrm{Mo}$ & $\mathrm{Cr}$ & $\mathrm{Fe}$ & $\mathrm{Zr}$ & $\mathrm{Sn}$ \\
at. $\%$ & balance & 8.8 & 2. & 2. & 0.8 & 2. & 0.8 \\
\hline
\end{tabular}

These experiments have shown that the composition of the $\alpha$ phase is quite independent of the temperature: thus, the $\alpha$ phase can be described as a stoichiometric compound. A great benefit can be drawn from this statement 
because the tie-lines are trivial. If this assumption appears very crude, it relies not only on our experimental measurements, but also on the following points. First, we have compared our measurements with the prediction of Saunders' database [3] input into Thermocalc software. This Calphad database encompasses the newest assessments of the thermodynamic parameters for titanium alloys $[4,5]$. Large differences between both volume fraction of $\alpha$ and equilibrium compositions have been found, especially for temperatures lower than $800^{\circ} \mathrm{C}$, i.e. in the range of the isothermal treatments that we have studied. These differences led to strong deviations of the overall kinetics from the experimental IT diagram. Then, the use of the Calphad description to provide realistic kinetics would have required an optimization of the fitting parameters (e.g. the activity coefficients as well as the interaction parameters), a task which was outside the aims of our study.

Second, our experimental evidence that the $\alpha$ phase composition does not vary much with temperature is not unique. This can be observed in the results of [6], where the variations in the compositions of $\mathrm{V}, \mathrm{Cr}, \mathrm{Fe}$ and $\mathrm{Al}$ (all elements present in the specific alloy studied) with temperature are very weak, and always lower than the reported experimental error bars.

Finaly, a quick look at a few isothermal sections in some ternary titanium alloy diagrams shows that the curvature of the $\beta$ phase field boundary is generally greater than that of the $\alpha$ phase field. Consequently, it is obvious that large variations in the equilibrium $\beta$ compositions correspond to small changes in the $\alpha$ equilibrium compositions, due to the fan-shaped distribution of the tie-lines.

Assuming that the $\beta$ phase is an ideal solution, the chemical equilibrium can be described with a solubility product [7]:

$$
\prod_{i}\left(x_{i}^{\beta^{\mathrm{eq}}}\right)^{x_{i}^{\alpha}}=K(T)
$$

where $x_{i}^{\alpha}$ and $x_{i}^{\beta^{\text {eq }}}$ are the equilibrium molar fractions of the species $i$ respectively in $\alpha$ and in $\beta$. Such a solubility product has been obtained from our measurements:

$$
K(T)=K_{0} \exp \left(-\frac{Q}{R T}\right)
$$

where $R$ is the gas constant, $K_{0}=0.819$ and $Q=3500 \mathrm{~J} / \mathrm{mol}$. The assessed values give a very good description of the equilibrium $\alpha$ phase volume fraction as a function of temperature [8]. This solubility product is further used to calculate the driving force for nucleation and to determine the composition of the parent phase at the interface during the growth processes.

Because some diffusion data were lacking in the literature, we have performed 
experiments with diffusion couples made of pure $\mathrm{Ti} / \beta$-Cez alloy, and determined the chemical diffusivities of the different species in the $\beta$ temperature range [8].

\section{Nucleation and growth laws}

\subsection{Transformation sequence}

Some experimental studies have identified the main successive mechanisms governing the formation of $\alpha_{\mathrm{GB}}$ and $\alpha_{\mathrm{WGB}}$ at high temperatures $[1,9,10]$. First, $\alpha_{\mathrm{GB}}$ nucleates heterogeneously on $\beta$ grain boundaries, and grows in directions normal to the grain boundaries. Second, as shown by systematic observations (Fig. 1b), some $\alpha_{\mathrm{WGB}}$ Widmanstätten plates appear along $\alpha_{\mathrm{GB}}$ allotriomorphs. Despite some observations in Ti-6.6 at.\% $\mathrm{Cr}$ and $\mathrm{Ti}-8.6$ at.\% Mo alloys which have proposed sympathetic nucleation [11], the specific appearance mechanisms of $\alpha_{\text {WGB }}$ are still unclear. Third, we have clearly established that the $\alpha_{\text {WGB }}$ lamellae grow inside the $\beta$ grains, gathered in colonies where they have identical crystallographic orientations. These observations are in agreement with previous studies $[12,13]$. The $\beta$ phase between the $\alpha$ lamellae is enriched in $\beta$ stabilizer species. Furthermore, Laude [1] showed that the $\beta$ phase is enriched in $\beta$ stabilizer species in front of the colonies, over short distances which decrease with the transformation temperature $\left(<10 \mu \mathrm{m}\right.$ at $790{ }^{\circ} \mathrm{C},<7 \mu \mathrm{m}$ at $750^{\circ} \mathrm{C}$ ). Consequently, the average composition of the $\beta$ matrix outside the colonies can be assumed not to change during the transformation.

Based on these experimental observations, the model describes the transformation with the following sequence (Fig. 2): (a) nucleation of $\alpha_{\mathrm{GB}}$ on a $\beta$ grain boundary; (b) growth of $\alpha_{\mathrm{GB}}$ on one side of a $\beta$ grain boundary; (c) appearance of $\alpha_{\mathrm{WGB}}$ at the growing $\alpha_{\mathrm{GB}}$ front; (d) growth of $\alpha_{\mathrm{WGB}}$ from $\alpha_{\mathrm{GB}}$.

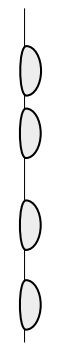

(a)

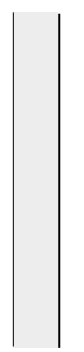

(b)

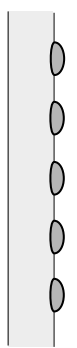

(c)

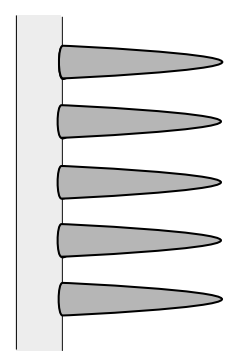

(d)

Fig. 2. Sequence of the phase transformation modelling for the high temperature morphologies: (a) nucleation of $\alpha_{\mathrm{GB}}$, (b) growth of $\alpha_{\mathrm{GB}}$, (c) appearance of $\alpha_{\mathrm{WGB}}$, (d) growth of $\alpha_{\mathrm{WGB}}$. 


\subsection{Nucleation of $\alpha_{\mathrm{GB}}$}

Despite the use of the pill box shape for describing the allotriomorph nucleation in some titanium based alloys [14], we have assumed that the $\alpha_{\mathrm{GB}}$ nuclei are spherical caps, in order to reduce the physical parameters involved in the model such as the interfacial energies, which are very difficult to assess experimentally.

The nucleation rate $\dot{N}_{n}$ of $\alpha_{\mathrm{GB}}$ (where $N_{n}$ is the number of nuclei and the dot denotes the time derivative) on the $\beta$ grain boundaries is obtained using the classical theory of nucleation [15]:

$$
\dot{N}_{n}=N \beta Z \exp \left(\frac{-\Delta G_{\text {het }}^{*}}{k T}\right) \exp (-\tau / t)=\dot{N}_{n_{s}} \exp (-\tau / t)
$$

where the different terms have their classical meaning and can be found in any standard textbook (see e.g. Ref. [20]); the only term specific to this study is the nucleation driving force expressed with the solubility product $K$ (Eq. 2) as $\Delta G_{v}^{\mathrm{c}}=R T / V_{m} \ln \left[\prod_{i}\left(x_{i}^{\beta^{0}}\right)^{x_{i}^{\alpha}} / K\right]$ where $V_{m}$ is the molar volume and $x_{i}^{\beta^{0}}$ the nominal molar fraction of element $i$ in the alloy.

\subsection{Growth of $\alpha_{\mathrm{GB}}$}

Based on the microstructural observations (Fig. 1a), the growth of $\alpha_{\mathrm{GB}}$ is assumed to be planar. Furthermore it is supposed to be controlled by the diffusion of the partitioning species. The width $W$ of the $\alpha_{\mathrm{GB}}$ layers is calculated by solving the conservation equations for each partitioning species in the $\beta$ matrix with the following boundary conditions (e.g. [17]).

(i) For an homogeneous molar volume, the balances for each partitioning species $i$ are:

$$
\left(x_{i}^{\beta^{*}}-x_{i}^{\alpha}\right) \dot{W}=J_{i}^{\beta^{*}}
$$

where $x_{i}^{\beta^{*}}$ is the molar fraction of species $i$ at the interface in $\beta$; and $J_{i}^{\beta^{*}}$ is the density of flux of species $i$ at the interface in $\beta$. For an ideal solution $J_{i}^{\beta}$ is simply given by the first Fick's law: $J_{i}^{\beta}=-D_{i}\left(\partial x_{i}^{\beta} / \partial z\right)$.

(ii) The assumption of local equilibrium at the interface is described by the solubility product:

$$
\prod_{i}\left(x_{i}^{\beta^{*}}\right)^{x_{i}^{\alpha}}=K(T)
$$


(iii) Moreover, the $\beta$ matrix is considered as an infinite medium. This is valid as long as there is no change in the composition of the matrix far from the interface (equal to the nominal composition $x_{i}^{\beta^{0}}$ ), i.e. as long as the diffusion fields induced by the growth of competing $\alpha_{\mathrm{GB}}$ do not overlap. This is reasonable for $\alpha_{\mathrm{GB}}$ because the order of magnitude of the diffusion lengths in front of the $\alpha_{\mathrm{GB}}$ allotriomorphs is much smaller than the size of the $\beta$ grains (a few hundred $\mu \mathrm{m})$.

The resulting equations to be solved are:

$$
\begin{gathered}
\prod_{i}\left[\left(x_{i}^{\beta^{0}}-x_{i}^{\alpha} \Omega_{i}\right) /\left(1-\Omega_{i}\right)\right]^{x_{i}^{\alpha}}=K(T) \\
\Omega_{i}=\mathcal{F}\left(\eta_{i}^{*^{2}}\right)=\sqrt{\pi} \eta_{i}^{*} \exp \left(\eta_{i}^{*^{2}}\right) \operatorname{erfc}\left(\eta_{i}^{*}\right)
\end{gathered}
$$

where $\Omega_{i}$ is the reduced supersaturation of the matrix in species $i \Omega_{i}=\left(x_{i}^{\beta^{*}}-\right.$ $\left.x_{i}^{\beta^{0}}\right) /\left(x_{i}^{\beta^{*}}-x_{i}^{\alpha}\right)$ and $\eta_{i}^{*}$ is the width $W$ scaled by the diffusion length of the species $i \eta_{i}^{*}=W /\left(4 D_{i} t\right)^{1 / 2}$.

\subsection{Appearance of $\alpha_{\mathrm{WGB}}$}

The appearance of $\alpha_{\mathrm{WGB}}$ is defined empirically because of the lack of knowledge concerning the mechanism involved. We have used the measurements of the final width of $\alpha_{\mathrm{GB}} W_{\mathrm{f}}$ as a function of temperature, realized by Laude [1]. A polynomial fit of the measurements in the temperature $\left[600{ }^{\circ} \mathrm{C}-890^{\circ} \mathrm{C}\right]$ gives:

$$
W_{\mathrm{f}}=7.98 \cdot 10^{-11} T^{2}-9.59 \cdot 10^{-8} T+2.93 \cdot 10^{-5}
$$

For temperatures lower than $600^{\circ} \mathrm{C}, W_{\mathrm{f}}$ is assumed to be the same as for $600{ }^{\circ} \mathrm{C}$ (i.e. $0.488 \mu \mathrm{m}$ ) because of the lack of measurements.

\subsection{Growth of $\alpha_{\mathrm{WGB}}$}

Because no data were available in the literature concerning the growth kinetics of $\alpha_{\mathrm{WGB}}$ in multicomponent $\beta$ metastable titanium alloys, we have measured the average $\alpha_{\text {WGB }}$ plates length on SEM images at different times of an isothermal treatment. A constant lengthening rate of $\dot{L}=4.33 \cdot 10^{-8} \pm 1.5 \cdot 10^{-8} \mathrm{~m} / \mathrm{s}$ is found for a transformation at $790^{\circ} \mathrm{C}$. This value compares well with that found experimentally in Ti-6.6 at.\% Cr by [14].

This observation supports the description of the $\alpha_{\mathrm{WGB}}$ diffusion controlled 
growth with a model accounting for the so-called point effect (e.g. see Ref. [19] and references therein). We have assumed that the plates are parabolic cylinders which grow by a diffusion controlled process. As for $\alpha_{\mathrm{GB}}$, the growth model relies on solving the conservation equations for each partitioning species in the $\beta$ matrix. These are expressed in the stationary moving parabolic coordinate frame [21,22] with the following boundary conditions: (i) Equation (4)balances at the interface for each partitioning species. (ii) The assumption of local equilibrium at the interface. It is also assumed that it is the diffusion at the very end of the plates, where the radius of curvature $\rho$ is the smallest, which controls the lengthening kinetics. Under these conditions, the equilibrium is expressed as follows:

$$
\prod_{i}\left(x_{i}^{\beta^{*}}\right)^{x_{i}^{\alpha}}=K(T) \exp \left(\frac{\gamma_{\alpha \beta} V_{m}}{\rho R T}\right)=K_{\rho}(T)
$$

(iii) The assumption that the $\beta$ matrix is an infinite medium for reasons similar to those put forward for $\alpha_{\mathrm{GB}}$. Indeed, the characteristic lengths of diffusion in front of the colonies are very small compared with the $\beta$ grain sizes. The resulting equations are (see e.g. Refs. [21,22]):

$$
\begin{aligned}
\prod_{i}\left[\left(x_{i}^{\beta^{0}}-x_{i}^{\alpha} \Omega_{i}\right) /\left(1-\Omega_{i}\right)\right]^{x_{i}^{\alpha}} & =K_{\rho}(T) \\
\Omega_{i} & =\mathcal{F}\left(P_{i}\right)
\end{aligned}
$$

where $P_{i}$ is the Péclet number of the plate edge $P_{i}=\rho \dot{L} /\left(2 D_{i}\right)$.

Contrary to the case of the planar growth, there are two characteristic lengths for describing the plates, i.e. the length related to the growth rate $\dot{L}$ on one hand, and the width related to the radius of curvature $\rho$ at the plate extremity on the other hand; thus, one more relation is necessary to solve the problem. When plotted against the radius of curvature, the velocity given by (9) reaches a maximum value. Zener [23] suggested the radius corresponding to this maximum velocity as the operating radius of the plates. Although empirical, this criterion proved to be quite successful for the Widmanstätten plates in other alloys (e.g. Refs. [24,25]). Equating to zero the partial derivative of (9) with respect to $\rho$ gives the necessary closure equation.

Finally, because it is difficult to assess precisely the interfacial energies, the value of $\gamma_{\alpha \beta}$ has been fixed such that the calculated velocities fit to the measured ones over a large temperature range, with $790^{\circ} \mathrm{C}$ as a guide: a value of $270 \mathrm{~mJ} / \mathrm{m}^{2}$ is obtained as the best fit. This value is in agreement with the nature of the semi-coherent interface which has been observed for that kind of morphology in binary titanium alloys $[18,26]$. 


\section{Global kinetics}

Microstructural observations have shown that the $\alpha_{\mathrm{GB}}$ amount depends greatly on the $\beta$ grain boundaries involved. This can be attributed to the difference in the $\alpha_{\mathrm{GB}}$ nucleation rates which are a function of $\gamma_{\beta \beta}$, i.e. the nature of the grain boundaries. To take into account such a scattering, it is necessary to introduce a statistical description of the transformation. For that purpose, we have considered one thousand tetrakaidecahedra (14-faced polyhedra) [27], representative of a polycrystalline $\beta$ phase. Comparisons with different numbers of $\beta$ grains have shown that one thousand is statistically sufficient, as well as computationally efficient for including the model in macroscopic mechanical computations of internal stresses [28]. All the tetrakaidecahedra have the same size, defined by the distance $D$ between opposite faces. In the present study, the value of $D$ is fixed to $D=200 \mu \mathrm{m}$, corresponding to the average diameter of the grains in the experimental samples obtained by image analysis. All the necessary geometric quantities, such as the volume and the surfaces are derived easily from $D$. This polycrystalline representation appears satisfactory when comparing the predicted specific surface $S_{v}=17800 \mathrm{~m}^{-1}$ for $D=200$ $\mu \mathrm{m}$ with the experimental one $S_{v}=16900 \pm 300 \mathrm{~m}^{-1}$.

A random wetting angle $\theta$ is allocated to each side of the tetrakaidecahedra faces within a Gaussian distribution (for simplicity) accounting for the scattering of the nature of the grain boundaries. The distribution parameters, i.e. the average value $\langle\theta\rangle$ and the standard deviation $\delta \theta$, are fitting parameters of the model. From preliminary calculations at several temperatures, these parameters have been set to the following values: $\langle\theta\rangle=19^{\circ}$ and $\delta \theta=15^{\circ}$. Thus we have $\gamma_{\beta \beta}(\langle\theta\rangle \mp \delta \theta) \in\left[500-540 \mathrm{~mJ} / \mathrm{m}^{2}\right]$ all values included in the range [500-900 mJ $\left./ \mathrm{m}^{2}\right]$ reported in Ref. [26] for titanium.

The implementation of the model is straightforward. All the faces (indexed $j \in$ $[1,14]$ ) of all the grains (indexed $k \in[1,1000]$ ) are treated sequentially at each time step in the following way. First, it is checked which transformation step is involved (Fig. 2). Then the appropriate microstructural variables are updated after having solved (3), (6) or (9): (i) The area covered by the $\alpha_{\mathrm{GB}}$ nuclei $S_{\mathrm{n}}^{j k}=\sum \pi\left(r_{j k}^{*} \sin \theta_{j k}\right)^{2}$ where the sum is performed over the total number $N_{\mathrm{n}}^{j k}$ of nuclei on the grain boundary $j$ of grain $k$, i.e. $N_{\mathrm{n}}^{j k}=\int \dot{N}_{n}^{j k} \mathrm{~d} t$. The duration from the begining of the treatment necessary for the $\alpha_{\mathrm{GB}}$ nuclei to cover completely the face area is called the wetting time $\Delta t_{\mathrm{w}}$. (ii) The width $W_{j k}$ of the $\alpha_{\mathrm{GB}}$ allotriomorphs layer. The corresponding transformed volume $V_{\mathrm{GB}}^{j k}=S_{\beta \beta}^{j k} W_{j k}$ can be deduced from it. (iii) Or the length $L_{j k}$ of the $\alpha_{\mathrm{WGB}}$ plates colony. The corresponding transformed volume $V_{\mathrm{WGB}}^{j k}=S_{\beta \beta}^{j k} L_{j k}$ is also computed. It must be emphazised that this transformed volume includes both $\alpha$ and $\beta$ phases; the plates themselves, the width of which is supposed to be equal to the size of the face; and in between, the $\beta$ phase enriched due to the 
thickening of these plates. For simplicity, it is assumed that both phases are in equilibrium inside the colonies. Then, the proportion of both phases is given by the lever rule.

For each transformation step, it is checked whether it must be changed into the next one, or whether it must be stopped when the $\alpha_{\text {WGB }}$ growth is concerned. The criteria for changing or stopping are: (i) Once $90 \%$ of the surface $S_{\beta \beta}^{j k}$ of the grain boundary $j$ involved is covered by the nuclei (i.e. $S_{\mathrm{n}}^{j k}=S_{\beta \beta}^{j k}$ ), the growth of the $\alpha_{\mathrm{GB}}$ allotriomorphs can start ${ }^{2}$. (ii) Once the width $W_{j k}$ of the $\alpha_{\mathrm{GB}}$ allotriomorphs layer has reached the experimental final width $W_{\mathrm{f}}$ given by (7), a new colony of $\alpha_{\text {WGB }}$ can start to grow from the whole surface of the $\alpha_{\mathrm{GB}}$ layer (equal to the $\beta$ grain boundary surface $S_{\beta \beta}^{j k}$ ). (iii) The $\alpha_{\mathrm{WGB}}$ growth is stopped in two cases: first, if the total transformed volume $\sum_{j}\left(V_{\mathrm{GB}}^{j k}+V_{\mathrm{WGB}}^{j k}\right)$ is equal to the volume of the grain $k$; secondly, if the length $L_{j k}$ of the colony is greater than the grain size $D$ minus the length of the colony growing from the opposite face of the same grain $k$, so as to prevent unphysical lengths of colony. (iv) The transformation is supposed to be finished when the transformation rate monitored by $\dot{\xi}$ is lower than $10^{-6} \mathrm{~s}^{-1}$, where $\xi$ is the transformed volume fraction $V_{\mathrm{t}} / V_{\mathrm{tot}}=\sum_{j, k}\left(V_{\mathrm{GB}}^{j k}+V_{\mathrm{WGB}}^{j k}\right) / V_{\mathrm{tot}}$.

Finally, we recall the microstructural input parameters used in the calculations in table 2 .

Table 2

Microstructural input parameters.

\begin{tabular}{lcccc}
\hline Parameters & $D(\mu \mathrm{m})$ & $\gamma_{\alpha \beta}\left(\mathrm{mJ} / \mathrm{m}^{2}\right)$ & $\langle\theta\rangle$ & $\delta \theta$ \\
\hline Values & 200 & 270 & 19 & 15 \\
\hline
\end{tabular}

\section{$5 \quad$ Results}

\subsection{Isothermal transformation kinetics}

The results of the calculations for an isothermal treatment at $790^{\circ} \mathrm{C}$ are shown in Fig. 3. The transformed volume fraction $\xi$ is plotted versus time in Fig. 3a. The corresponding microstructural parameters are plotted versus time in Fig. 3b: the average number of allotriomorphs layers per grain, the average number

$\overline{2}$ Neglecting the incubation time, the integration of (3) gives a infinite time for exhausting all the nucleation sites. This result is unrealistic because the nuclei are able to grow along the grain boundaries, decreasing the number of nucleation sites. The $90 \%$ criterion is a first way to account for this process. Some additional work is under process to further analyse the wetting of the grain boundaries by allotriomorphs. 
of colonies per grain, and the number of active grains where the transformation is progressing (i.e. where $\sum_{j} \dot{V}_{\mathrm{WGB}}^{j k}>0$ ).

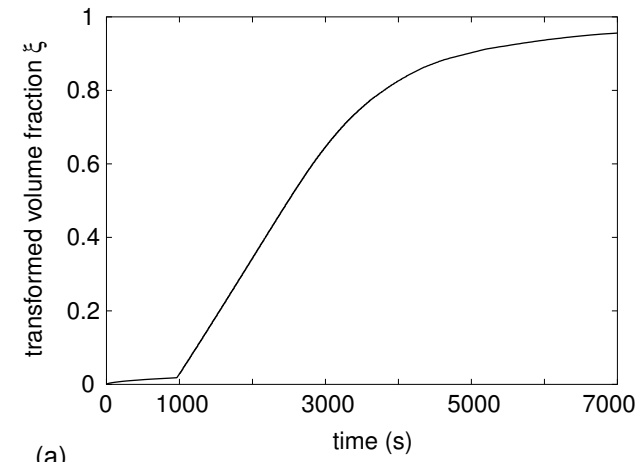

(a)

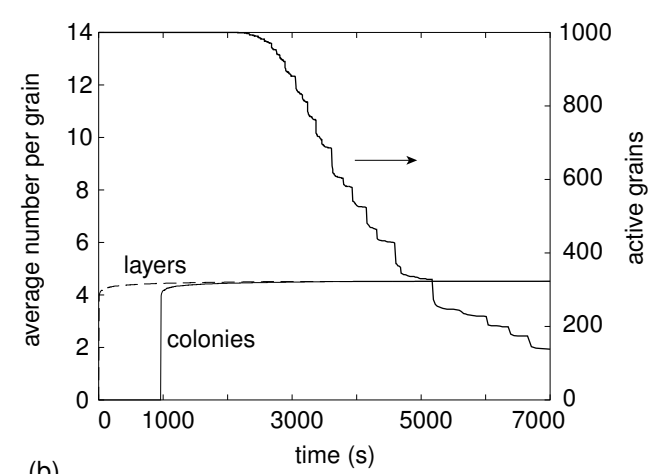

(b)

Fig. 3. Isothermal treatment at $790{ }^{\circ} \mathrm{C}$ : (a) transformed volume fraction $\xi$ vs. time; (b) average numbers of $\alpha_{\mathrm{GB}}$ allotriomorph layers and of $\alpha_{\mathrm{WGB}}$ colonies per grain, and number of active grains vs. time.

The evolution of $\xi$ can be split into two stages. During the first one, up to about $1000 \mathrm{~s}$, a small increase is observed. It corresponds to the growth of $\alpha_{\mathrm{GB}}$ alone, as shown in Fig. 3b: the number of allotriomorph layers increases abruptly from the very begining and then slowly, whereas the number of $\alpha_{\text {WGB }}$ colonies is null up to about $1000 \mathrm{~s}$. Thus, $\xi$ is nearly proportional to $\sqrt{t}$, because the number of layers is quite constant, and because all the layers follow the same growth law $W=C \sqrt{t}$ with the same proportionality factor $C$. This stage ends abruptly when the width of a majority of the $\alpha_{\mathrm{GB}}$ layers reaches the empirical value $W_{\mathrm{f}}$. At the beginning of the second stage, there is a sharp increase in the number of $\alpha_{\mathrm{WGB}}$ colonies, which is simply the translation of the sharp increase of the number of allotriomorph layers at the begining of the treatment.

During the second stage, the growth of the $\alpha_{\mathrm{WGB}}$ colonies induces a rapid increase in the transformed volume fraction. Indeed, because the lengthening rate is time independent, the rate of transformation $\dot{\xi}$ is constant for a constant number of growing colonies. The slight convexity of $\xi(t)(\ddot{\xi}>0)$ observed during the begining of this second stage (1000 $\mathrm{s} \leq t \leq 2500 \mathrm{~s})$ is due to the slight increase in the number of colonies.

For $t>2500 \mathrm{~s}, \dot{\xi}$ slows down progressively $(\ddot{\xi}<0)$ when the number of active grains starts to diminish. At the end of the transformation at $790^{\circ} \mathrm{C}$, the average number of colonies per grain is about five, lower than the maximum value imposed by the geometry of the tetrakaidecahedra.

In Fig. 4a, we have compared the global kinetics with the experimental in-situ measurements by electrical resistivity [1]. Because of the geometrical constraints, the value reached by the transformed volume fraction $\xi$ may be less than unity at the end of the transformation (i.e. when $\dot{\xi}<10^{-6} \mathrm{~s}^{-1}$ ). Thus, we have plotted $\xi^{\prime}$, i.e. $\xi$ normalized by the transformed volume at the end of the transformation. The overall agreement is good. It must be emphasized that 


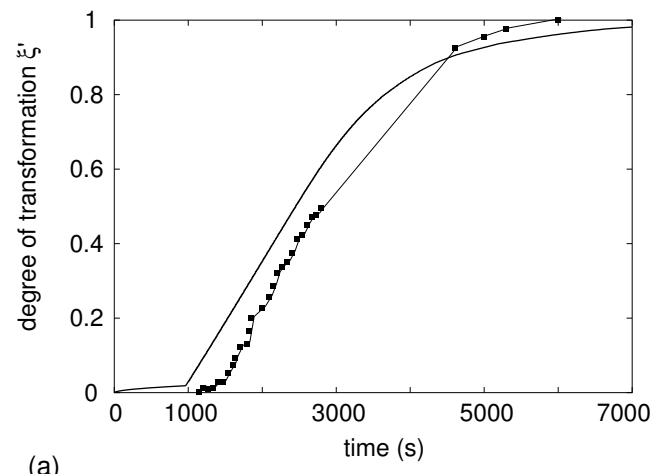

(a)

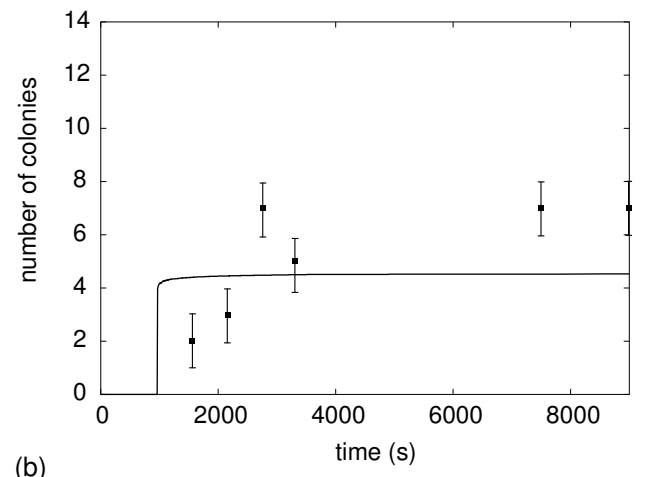

(b)

Fig. 4. Comparison with experimental results at $790{ }^{\circ} \mathrm{C}$ [1]: (a) degree of transformation $\xi^{\prime}$ vs. time; (b) average numbers of $\alpha_{\mathrm{WGB}}$ colonies per grain vs. time. Continuous lines are for the calculations and dots for the measurements.

the duration of the first stage is close to the so-called incubation time of the order of $1000 \mathrm{~s}$ deduced from the experimental measurements. Usually, the experimental incubation time is attributed to the transient nucleation process (i.e. $\tau$ in (3)). But, at $790^{\circ} \mathrm{C}, \tau<1 \mathrm{~s}$ for wetting angles $\theta<45^{\circ}$. Moreover, as stated above, during this first stage the degree of transformation is only a few per cent which is underneath the experimental uncertainty at the beginning of the measurements. Thus, it is reasonable to attribute the so-called experimental 'incubation time' to the growth of the $\alpha_{\mathrm{GB}}$ rather than to the nucleation process. Additional experiments were performed and have confirmed this result.

In Fig. 4b, we have compared the number of colonies vs. time predicted by the model and the measurements of Laude [1]. The predicted evolution is quicker and more sudden than the experimental one. Moreover, the calculated number of colonies at the end of the transformation is smaller than what has been measured, even when considering the experimental uncertainties of two colonies.

To explain the time evolution of the $\alpha_{\mathrm{GB}}$ layers $/ \alpha_{\mathrm{WGB}}$ colonies, we have plotted in Fig. 5 the wetting times $\Delta t_{\mathrm{w}}$ vs. the corresponding wetting angles $\theta$ at $790^{\circ} \mathrm{C}$. Two domains of $\theta$ are clearly separated by the angle $\theta_{c}$ which is related to the duration of the transformation $\Delta t_{\text {tot }}$ at $790^{\circ} \mathrm{C}$ (dashed lines). For $\theta<\theta_{\mathrm{c}}, \Delta t_{\mathrm{w}}$ is small when compared to $\Delta t_{\text {tot }}$. In contrast, for $\theta \geq \theta_{\mathrm{c}}$, $\Delta t_{\mathrm{w}}>\Delta t_{\mathrm{tot}}$. Hence, as soon as a treatment starts, the faces with $\theta<\theta_{\mathrm{c}}$ are immediately wetted. Then, the number of $\alpha_{\mathrm{GB}}$ layers increases suddenly. Only a few faces with $\theta \approx \theta_{\text {c }}$ are wetted during the rest of the transformation; a slight increase in the number of $\alpha_{\mathrm{GB}}$ layers is then observed.

In Fig. 5, we have also plotted the distribution of the wetting angles for the Gaussian centered around $\langle\theta\rangle=19^{\circ}$ with $\delta \theta=15^{\circ}$. The proportion of the wetted faces, and so of the $\alpha_{\mathrm{WGB}}$ colonies at the end of the transformation corresponds to the shaded area divided by the total area under the Gaussian. 


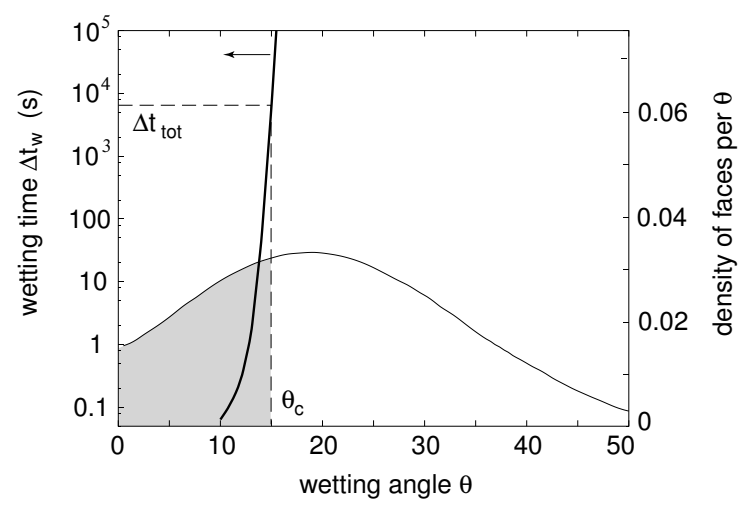

Fig. 5. Wetting time and density of faces per angle vs. wetting angle at $790^{\circ} \mathrm{C}$.

\subsection{Influence of the temperature}

The influence of the temperature is shown in figure 6. Concerning the first step, the higher the temperature, the longer is the first stage of transformation. It can also be noticed that the volume fraction of the $\alpha$ phase at the end of the first stage is the same whatever the temperature is, because the empirical criterion used for predicting the appearance of $\alpha_{\mathrm{WGB}}$ does not lead to large variations in the total volume of $\alpha_{\mathrm{GB}}$ [1]. Nonetheless, the slight increase of the final width $W_{\mathrm{f}}$ with temperature is sufficient to lengthen the first stage as the temperature increases because $W \propto \sqrt{t}$.

Similarly, during the second stage, the higher the temperature, the slower is the transformation rate. This is related to the number of colonies and to the lengthening rate $\dot{L}$ which both decrease with increasing temperatures, above $730{ }^{\circ} \mathrm{C}$.

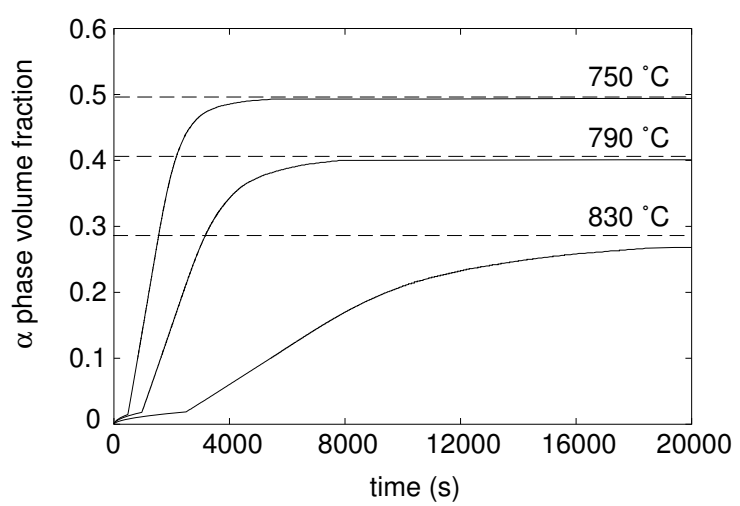

Fig. 6. $\alpha$ volume fraction vs. time for different temperatures. The dashed lines are the equilibrium $\alpha$ phase volume fractions.

Lastly, for the microstructural parameters chosen (Table 2), whatever the temperature is, the final volume fraction of $\alpha$ is slightly below the equilibrium one. This is a consequence of the incomplete fill-in of the grains by the colonies due to the geometrical constraints on their maximal lengths. 
These calculated results are compared to experimental transformation kinetics obtained by electrical resistivity [1] for 750,790 and $830^{\circ} \mathrm{C}$ in Fig. 7a. The overall agreement is good. One can therefore perform additional calculations under isothermal conditions to construct the IT diagram of the $\beta$-Cez alloy (Fig. 7b). The calculated $10 \%$ and $90 \%$ of transformation are in good agreement with the experimental measurements in the high-temperature range. For lower temperatures $\left(\leq 700^{\circ} \mathrm{C}\right)$, the transformation kinetics obtained experimentally is clearly faster than what is predicted by the model. Indeed, the discrepancy is related to the appearance of a thin intragranular Widmanstätten structure $\left(\alpha_{\mathrm{WI}}\right)$.

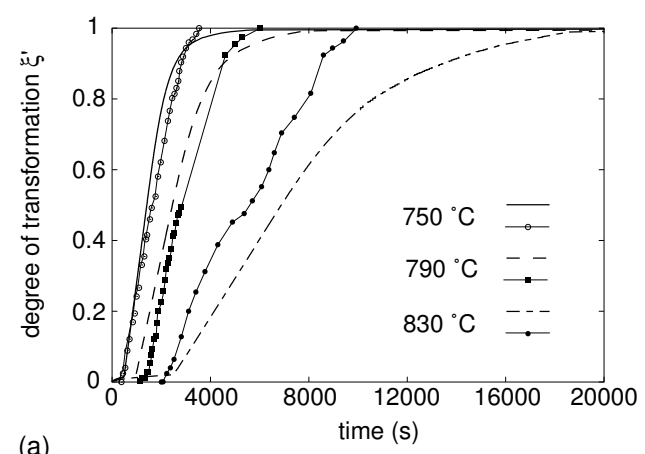

(a)

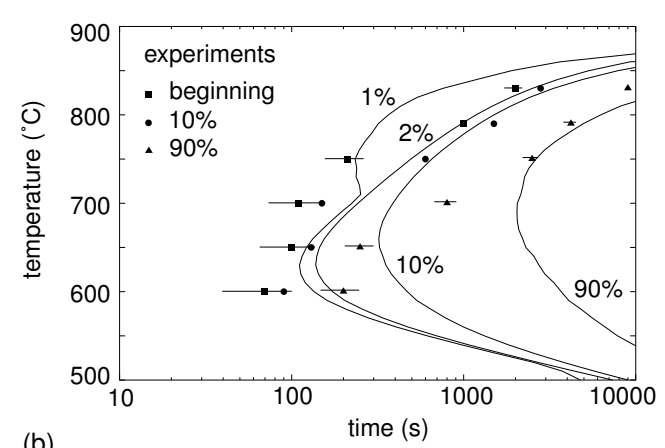

(b)

Fig. 7. Comparison with experiments: (a) global kinetics for 750,790 and $830^{\circ} \mathrm{C}$; (b) IT diagram of $\beta$-Cez in the high temperatures range. The lines are for the calculations and the dots for the experiments. For clarity, the error bars have been omitted for the experimental $10 \%$ of transformation.

Concerning the beginning time of transformation, the comparison is more complicated due to the uncertainty in the experimental measurements which ranges from typically $\approx 200 \mathrm{~s}$ at the highest temperatures to typically $\approx 30$ $\mathrm{s}$ at the lower ones. In Fig. $7 \mathrm{~b}$, we have reported the calculated $1 \%$ and $2 \%$ of transformation. These curves show that, in the high-temperature range $(>$ $700{ }^{\circ} \mathrm{C}$ ), the first $3 \%$ of transformation span large time ranges in which the experimental beginning times of transformation are included. These large ranges can be explained by the change in the growth process between $1 \%$ and $2 \%$ of transformation: only $\alpha_{\mathrm{GB}}$ is predicted to grow at $1 \%$ of transformation, while $\alpha_{\text {WGB }}$ is at $2 \%$. Hence, the overall agreement between the model and the experiments can be considered as very satisfactory. Moreover, the model predicts the formation of $\alpha_{\mathrm{GB}}+\alpha_{\mathrm{WGB}}$ morphologies at temperatures where a majority of $\alpha_{\mathrm{WI}}$ morphology appears. Experimental observations confirmed the occurence of these morphologies for the lower temperatures of 600 and $650{ }^{\circ} \mathrm{C}$.

Besides the global kinetics, microstructural parameters can be predicted by the model. In Fig. 8 we have reported the average number of colonies per grain at the end of the transformation as a function of the temperature. The final number of colonies increases when the transformation temperature de- 


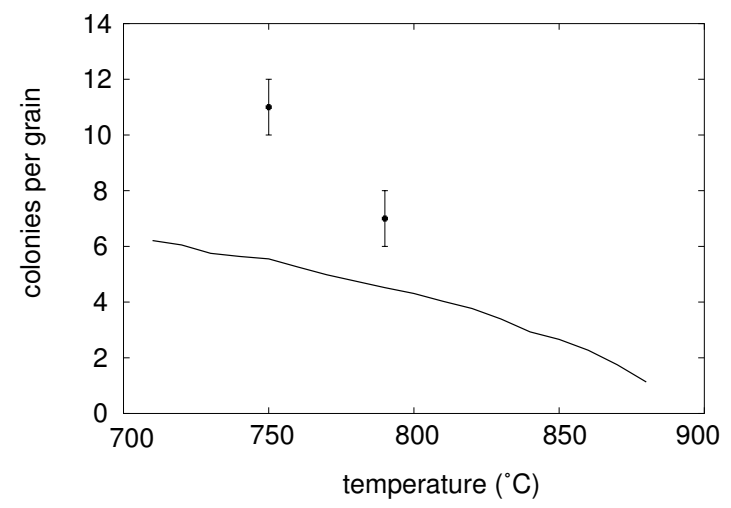

Fig. 8. Average final number of colony vs. temperature.

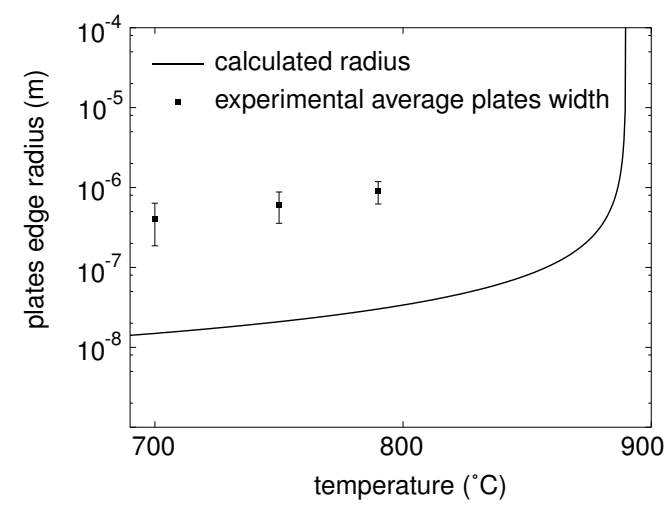

Fig. 9. Calculated plates edge radius vs. temperature compared with experimental plates width.

creases. Indeed, as the temperature decreases, the driving force for nucleation increases: the number of $\beta$ grain boundaries which are wetted by $\alpha_{\mathrm{GB}}$ layers increases as well, as do the number of colonies which have grown from $\alpha_{\mathrm{GB}}$. As shown in Fig. 8, these predictions agree qualitatively with the experimental observations. Nonetheless the predictions are lower than the measurements. In Fig. 9, we have compared the computed plate-edge radius with their experimental widths determined on SEM images with an overall uncertainty of about $150 \mathrm{~nm}$, at different temperatures. The same trend is observed for both: they increase as the transformation temperature does. Furthermore, a factor of about 30 between the width and the radius is found whatever the temperature is.

\subsection{Influence of parameters sensitive to a prior deformation}

The thermal and mechanical treatments designed for these titanium alloys often start with a deformation step (e.g. forging). During this step, subgrains are formed that dynamic or metadynamic recrystallization can change into grains with small sizes, resulting in a grain refinement as well as a broadening 
of the grain boundary energy distribution [29]. The microstructural parameters of the model which can take into account these effects are the distribution of the wetting angle $\theta$, and the grain size $D$.

First, we have changed the width of the Gaussian distribution around the average value $\langle\theta\rangle=19^{\circ}$. The time evolutions of $\xi$ resulting from the calculations performed with different values of the standard deviation $\delta \theta$ are compared in Fig. 10. Two ranges of $\delta \theta$ are observed with opposite behaviours:

for $\delta \theta<10^{\circ}$, when $\delta \theta$ increases the global kinetics accelerate mainly because the $\alpha_{\text {WGB }}$ growth stage is accelerated;

for $\delta \theta>10^{\circ}$, increasing $\delta \theta$ induces a small slow down of the $\alpha_{\mathrm{WGB}}$ growth stage.

For explaining this two-fold behaviour, the growth kinetics of the different morphologies can be discarded because they do not change for a constant temperature. On the contrary, this behaviour is closely related to the wetting of the faces which controls the number of the growing colonies (Fig. 11).

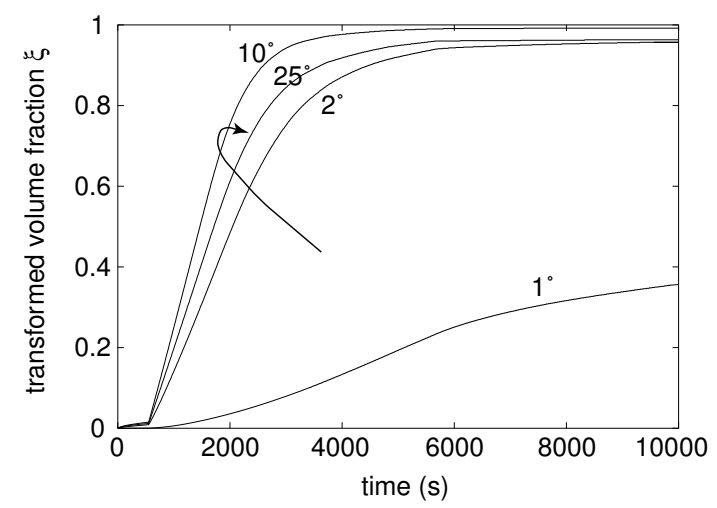

Fig. 10. Time evolution of $\xi$ for different standard deviations $\delta \theta$ at $790{ }^{\circ} \mathrm{C}$. The arrow follows the increasing $\delta \theta$

For very small $\delta \theta$ (e.g. $\delta \theta=1^{\circ}$ in Fig. 10), many of the faces are close to the critical wetting angle $\theta_{\mathrm{c}}$ : the appearance of new colonies is progressive and so is the transformed volume fraction, leading to very low transformation kinetics.

For moderate $\delta \theta \leq 10^{\circ}$, enlarging the Gaussian increases the number of faces which are rapidly wetted and thus increases the number of $\alpha_{\mathrm{WGB}}$ colonies (Fig. 11). This increase leads to a split of the global kinetics into the two identified growth stages as well as an acceleration of the $\alpha_{\text {WGB }}$ growth stage.

For large $\delta \theta>10^{\circ}$, a part of the Gaussian spans unphysical negative values of $\theta$. These values are then discarded and new positive values following again a Gaussian distribution are allocated to the corresponding faces. The resulting distribution is the sum of two Gaussians, the area of the second one being the area of the part of the first one with the negative $\theta$. This results in a skew distribution where the percentage of wetted faces is lower than for a symmetric Gaussian. 


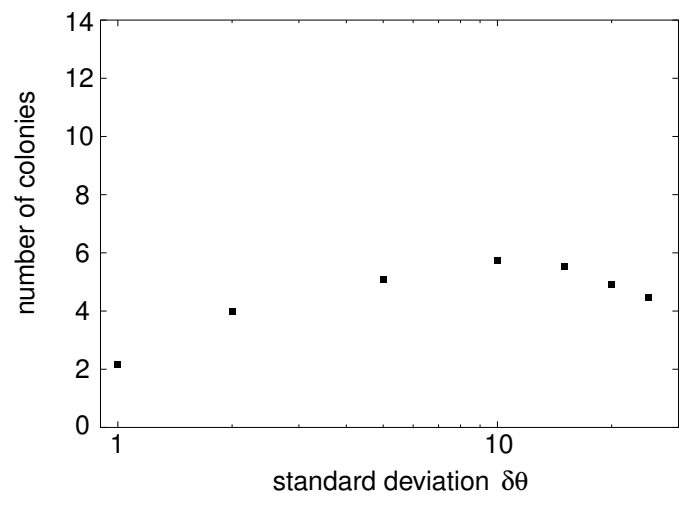

Fig. 11. Number of $\alpha_{\mathrm{WGB}}$ colonies vs. the standard deviations $\delta \theta$.

The influence of the grain size is illustrated in Fig. 12. We have compared the global kinetics for different grain sizes $D(100,200$ and $400 \mu \mathrm{m})$, keeping the other microstructural parameters equal to those of Table 2. A global acceleration is observed when the grain size diminishes. Moreover, the final transformed volume fraction $\xi$ decreases when the grain size increases.

The duration of the $\alpha_{\mathrm{GB}}$ growth is independent of the grain size because changing $D$ does not change the final width $W_{\mathrm{f}}$ of the $\alpha_{\mathrm{GB}}$ layers. However, the total volume fraction of $\alpha_{\mathrm{GB}}$ is larger for smaller grain sizes due to the larger specific surface of grain boundaries.

Concerning the $\alpha_{\mathrm{WGB}}$ formation, changing the grain size has no effect on the growth rates $\dot{W}$ and $\dot{L}$ because the enrichment/depletion of the matrix is not taken into account. Moreover, in the present case, there is no effect on the number of colonies because the distribution of wetting times $\Delta t_{w}$ is only a function of the temperature. Thus the rate $\dot{\xi}=\dot{V}_{\mathrm{t}} / V_{\text {tot }} \propto 1 / D$. However, for smaller grain sizes, it can be expected that the earlier growth of a few colonies may lead to an earlier complete filling of the grain volume, reducing the number of colonies per grain.

Finally, the fact that the value of $\xi$ reached at the end of the transformation depends on the grain size is quite unexpected. At first glance, this feature could be attributed to the adjustment of the matrix composition as transformation proceeds. But, even for large fractions of $\alpha$ phase, the average composition of the matrix does not vary much: first because the contribution of the allotriomorphs is very weak (at most $2 \%$ of the total volume is transformed at the grain boundaries); and secondly because the colonies of the Widmanstätten structure are composed of both phases with the respective equilibrium compositions. This last assumption is very reasonable because the diffusion length is of the order of the width of the $\alpha$ plates, i.e. at most a few $\mu \mathrm{m}$ (Fig. 9). Then, it can be safely assumed that the composition of the $\beta$ phase in between the plates adjusts very quickly to reach the equilibrium, while the average matrix composition ahead of the colonies' advancing front is barely changed (in a similar way to what can happen in front of eutectoid colonies). Then the soft impingement of the colonies plays a very minor role in stopping the transformation, even if it could smooth the end of the transformation curves. For 
isothermal treatments, taking into account the change in the matrix composition is not relevant to explaining that the final $\xi$ depends on the grain size. In fact, the reason why the model predicts such a dependency is the geometrical constraints that we imposed on the colonies. First, recall that we have considered constraints on their lengths as necessary for a good assessment of the mechanical properties. Indeed, the colonies could have been grown until the whole space was filled. Nonetheless, in that case, the colony lengths could reach a few grain diameters, which is not observed experimentally and which is problematic for assessing mechanical properties from the microstructure sizes. When the colonies are stopped for geometric reasons, untransformed 'dead zones' can remain if only a few faces of the grains have been wetted by the allotriomorph layers. The unwetted faces have very small nucleation potential due to their unfavorable energy. Some driving force can thus be still available for the transformation to proceed. If the stopping criterion on the rate of transformation is removed, the transformation proceeds at a rate controlled by nucleation on the unfavorable faces. This rate is so slow that the time for completion of the transformation is far greater than the duration of a typical experiment (several orders of magnitude larger). Because this dead-zone effect is accentuated as the grain size increases, the equilibrium fraction is all the more difficult to reach since the $\beta$ grains are large. It must be noted that experimental results have shown the formation of fine $\alpha$ grains in such dead zones inherited from slow continuous cooling [30]. The fact that the colonies keep the same size fixed by the tetrakaidecahedron grain geometry is the second geometrical constraint which contributes to the existence of dead zones. The next step to improve the modelling of such transformation sequences would be to take into account nucleation at the edges and corners. This has not been done yet because more experimental evidence for the relationship between the shapes of the colonies and the places where they nucleate are needed.

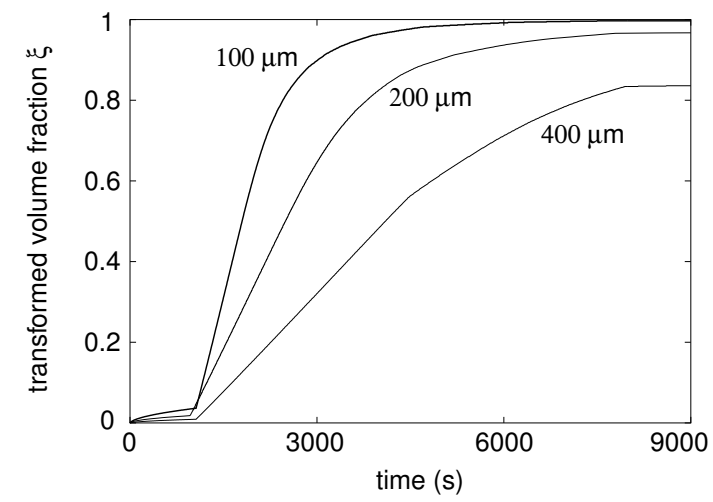

Fig. 12. Degree of transformation $\xi$ vs. time for different grain sizes $D$.

In conclusion, it must be stressed that the previous results relative to both parameters $\delta \theta$ and $D$ are still in qualitative agreement with the experimental in-situ measurements of the global transformation kinetics performed by Laude [1] after a prior deformation in the $\beta$ phase field. Indeed, these exper- 
iments have shown that the kinetics are accelerated by a prior deformation. This agreement seems to support the relevance of our model. More work is currently in progress to assess quantitatively the change in the parameters of the model resulting from a prior deformation.

\section{Conclusions}

We have developed a model able to predict the kinetics of $\alpha_{\mathrm{GB}}$ and $\alpha_{\mathrm{WGB}}$ morphologies during isothermal treatments in some titanium alloys. It is based on nucleation and growth laws, and the necessary data are the thermodynamic description of the phases, the diffusion coefficients, and some structural parameters such as the interface energy, the grain boundary energies distribution and the average grain size.

This model has been applied to the $\beta$-Cez alloy. The calculated overall transformation kinetics are in good agreement with experimental measurements. Moreover, the evolution of some microstructural parameters, which are important for the mechanical properties such as the mean number of colonies per grain, follows the same trends as observed experimentally.

Finally, provided that the thermodynamic description of the phases is available, this model can easily be used for other titanium alloys where similar sequences of morphologies are observed. Furthermore, it can take into account the effect of a prior deformation of the $\beta$ phase which is known to modify the nucleation conditions.

\section{References}

[1] Laude E, Gautier E, Archambault P, Denis S. Revue Métall 1996:1067.

[2] Héricher L, Gautier E, Ledeuil JB. Revue Métall 2000:113.

[3] Saunders N. In: Proceedings of the $8^{\text {th }}$ World Conference on Titanium. Birmingham, UK: Blenkinsop, PA; 1995. p. 2167.

[4] Kaufman L. Calphad 1977;1:7. Calphad 1978;2:55-82-117. Kaufman L, Nesor H. Calphad 1978;2:295-325.

[5] Onodera H, Ohno K, Yamagata T, Yamazaki M. Trans ISIJ 1988;28:803.

[6] Enomoto M, Yoshida T, ISIJ Int 1991;31:767.

[7] Lupis CHP. Chemical Thermodynamics of Materials. New York, NY: NorthHolland; 1983. p. 117.

[8] Héricher L. PhD thesis, INPL, Nancy; 2004. 
[9] Bein S. PhD thesis, CNAM, Paris; 1996.

[10] Angelier C. PhD thesis, CNAM, Paris; 1993.

[11] Sarath Kumar Menon E, Aarsonson HI. Acta Metall 1987;35:549.

[12] Chaussy-Mraizika F. PhD thesis, INPG, Grenoble; 1996.

[13] Mora L. PhD thesis, Paris XI; 1994.

[14] Sarath Kumar Menon E, Aarsonson HI. Metall Trans A 1986;17:1703.

[15] Russel KC. Adv Coll Interf Sci 1980;13:205.

[16] Russel KC. Acta Metall 1968;16:761.

[17] Bourne JP, Atkinson C, Reed RC. Metall Mater Trans A 1994;25:2683.

[18] Sarath Kumar Menon E, Aarsonson HI. Acta Metall 1986;34:1963.

[19] Hillert M, Höglund L, Agren J. Acta Mater 2003;51:2089.

[20] Christian JW. Theory of Transformations in Metals and Alloys - Part I, $2^{d}$ ed. London: Pergamon Press; 1975. p. 181.

[21] Ivantsov GP. translation of: Dokl Akad Nauk SSSR 1947;58:567.

[22] Horvay G, Cahn JW. Acta Metall 1961;9:695.

[23] Zener C. Trans AIME 1946;167:550.

[24] Simonen EP, Aaronson HI, Trivedi R. Metall Trans A 1973;4:1239.

[25] Gupta SP. Z Metallkd 1999;90:182.

[26] Plitcha MR, Perepezko JH, Aaronson HI, Lange WF. Acta Metall 1979;28:1031.

[27] Cahn JW. Acta Metall 1956;4:449.

[28] Da Costa Teixeira J, Héricher L, Appolaire B, Aeby-Gautier E, Cailletaud G, Denis S, Spath N. In: Proceedings of the International Conference on Thermal Process Modelling and Computer Simulation. Nancy, France: Denis, S; 2003.

[29] Furuhara T, Toji Y, Abe H, Maki T. Mater Sci Forum 2003;426-432:655.

[30] Angelier C, Bein S, Béchet J. Metall Mater Trans A 1997;28:2467. 